\title{
Synthesis and Characterization of La-Doped Luminescent Multilayer Films
}

\author{
Tianlei Wang, Meitang Liu, Hongwen Ma, and Defu Cao \\ Beijing Key Laboratory of Materials Utilization of Nonmetallic Minerals and Solid Wastes, National Laboratory of Mineral Materials, \\ School of Materials Science and Technology, China University of Geosciences, Beijing 100083, China
}

Correspondence should be addressed to Meitang Liu; mtliu@cugb.edu.cn and Hongwen Ma; mahw@cugb.edu.cn

Received 17 July 2016; Revised 8 November 2016; Accepted 30 November 2016; Published 22 January 2017

Academic Editor: José L. Arias Mediano

Copyright (C) 2017 Tianlei Wang et al. This is an open access article distributed under the Creative Commons Attribution License, which permits unrestricted use, distribution, and reproduction in any medium, provided the original work is properly cited.

In this work, we have successfully designed ordered luminescent multilayer films based on La-doped nonmagnetic or magnetic inorganic nanostructure with electronic microenvironment (EM). The inorganic nanosheets with opposite charge can assemble EM between the interlayers. At the same time, their elements on nanosheets of layer double hydroxides (LDHs) are facile to be replaced so that we can introduce transition metal or lanthanide elements. Besides, ferromagnetic effect (FE) can be formed in this microenvironment due to introducing transition metal on LDHs nanosheets. As a result, we confirm that EM, FE, and doping La element in the LDHs can affect the vibration of backbone of chromophores and then prolong the luminescent lifetime, which suggests a new pathway for developing the novel light-emitting thin films.

\section{Introduction}

Nowadays, an attractive target has been focused on which is layered materials for both fundamental research and practical application because of their intrinsic unique twodimensional structure, rich physics, and chemical properties [1-3]. Specifically, the nanosheets of those have been used as structural units in multifunctional construction. Because of the flexibility of composition (nanosheets and guest between interactions), such materials have attracted tremendous attention for their potential applied value $[4,5]$. Due to the successful achievement of the monolayer or few nanosheets about layered materials, the layered materials have been serving as functional units to build ordered thin films via layer-by-layer self-assemble method (LBL method) [6-9]. Recently, the scientists assembled successfully ordered and regular multifunctional thin films, such as luminescent films, magnetic films, and even biological films [10-16].

With the urgent targets to deal with the crisis of energy depletion, enthusiastic exploration of the environmental and efficient energy materials are engaged. So luminescent materials based on lanthanide elements have been candidates in order to be applied in optoelectronic devices and optical communications $[17,18]$. Series of matrix phosphors doped with various lanthanide ions were obtained and adjusted the element content in order to be applied for white LEDs $[19,20]$. A sensitizer was assembled by lanthanide complexes pillaring in inorganic layered materials between the interlayer, and it has attracted tremendous attention for practical device applications [21]. On the other hand, the incorporation of lanthanide ions on the layered materials' nanosheets has been reported to achieve visible or infrared luminescence, respectively [22].

Layered double hydroxides (LDHs), as a type of significant host-guest materials, have been considered as a fascinating candidate due to their simple preparation method, environmental protection, and excellent chemical stability [2326]. At the same time, isomorphous replacement of certain $\mathrm{M}^{2+}$ cations by $\mathrm{M}^{3+}$ cations in $\mathrm{LDHs}$, such as $\mathrm{Al}^{3+}$ and $\mathrm{Ga}^{3+}$, gives positively charged layers, and montmorillonite's (MMT) nanosheets also process opposite charge due to different isomorphic substitution compared with LDHs [26, 27]. In our previous work, it was testified that electronic microenvironment (EM) assembled by LDHs and MMT nanosheets can prolong the luminescent lifetimes of thin films [28]. Besides, when introducing transition metal on nanosheets, LDHs can be served as ferromagnetic layers. As a result, we succeeded 
in obtaining ordered thin films with ultraprolonged lifetimes and verified that ferromagnetic effect (FE) can also enhance luminescent lifetimes of chromophores in coordination with EM [29]. Herein, in this paper, we synthesized the La-doped magnetic or nonmagnetic LDHs and assembled the luminescent thin films via LBL mothed. By rationally choosing the inorganic nanosheets; we proved that the EM and FE can be beneficial for the optical properties of those luminescent thin films based on La-doped LDHs. Therefore, this work put forward a viable way for fabricating ordered La-doped inorganic nanostructure with EM and FE, which can provide better chance for the application of the next generation of optical devices.

\section{Experimental Section}

2.1. Reagents and Materials. $\mathrm{Mg}\left(\mathrm{NO}_{3}\right)_{2} \cdot 6 \mathrm{H}_{2} \mathrm{O}, \mathrm{Al}\left(\mathrm{NO}_{3}\right)_{3}$. $9 \mathrm{H}_{2} \mathrm{O}, \mathrm{Co}\left(\mathrm{NO}_{3}\right)_{2} \cdot 6 \mathrm{H}_{2} \mathrm{O}$, and $\mathrm{La}\left(\mathrm{NO}_{3}\right)_{3} \cdot 6 \mathrm{H}_{2} \mathrm{O}$ were all supplied by the Xilong Chemical Plant. $\mathrm{NaOH}, \mathrm{NH}_{3} \cdot \mathrm{H}_{2} \mathrm{O}, \mathrm{H}_{2} \mathrm{O}_{2}$ (30\%), and $\mathrm{H}_{2} \mathrm{SO}_{4}(95 \%-98 \%)$ were purchased from Beijing Chemical Reagent Company. Bis(N-methylacridinium) (BNMA) was supplied by J\&K Scientific, Ltd. Na-montmorillonite (MMT) was supplied by Zhejiang Feng Hong New Materials Co., Ltd., and polyvinyl alcohol (PVA, DP = $1750 \pm 50$ ) was manufactured by Tianjin Fuchen Chemical Reagent Plant.

2.2. Characterization. Double beam UV-vis spectrophotometer (TU-1901) was used to monitor the process of the depositing cycles. Fluorescence spectrophotometer (F-4600, Hitachi, Japan) was used to perform the luminescent property of samples. Edinburgh Instruments' steady and transient time-resolved fluorescence spectrometer was used to test the fluorescence decay, and the $375 \mathrm{~nm}$ pulse laser radiation was used as the excitation source.

2.3. Synthesis of La-Doped LDHs. The La-doped LDHs were synthesized via coprecipitation method, similar to our previous work [28]. Typically, divalent metallic cations $\mathrm{M}^{2+}\left(\mathrm{Mg}^{2+}\right.$ or $\left.\mathrm{Co}^{2+}\right)$ and trivalent metallic cations $\mathrm{M}^{3+}\left(\mathrm{Al}^{3+}, \mathrm{La}^{3+}\right)$ were mixed in boiled deionized water $\left(\mathrm{M}^{2+} / \mathrm{M}^{3+}=2\right.$ and $\mathrm{Al}^{3+} / \mathrm{La}^{3+}$ $=1$ ). Certain amount of solution of sodium hydroxide was added into the salts solution under ongoing stirring. Mixed solutions were stirred at $80^{\circ} \mathrm{C}$ for $24 \mathrm{~h}$ under $\mathrm{N}_{2}$ gas, and then the precipitate was centrifuged and dried at $60^{\circ} \mathrm{C}$.

\subsection{Exfoliation of MgAlLa-LDHs, CoAlLa-LDHs, and MMT.} $1 \mathrm{~g}$ MMT was added in $1 \mathrm{~L}$ deionized water with continuous stirring for $28 \mathrm{~d}$. And then the solution with exfoliated MMT nanosheets was obtained by centrifuging the suspension at $10000 \mathrm{rpm}$ for $10 \mathrm{~min}$. The positively charged MgAlLaLDHs and CoAlLa-LDHs nanosheets were obtained by $0.1 \mathrm{~g}$ MgAlLa-LDHs or CoAlLa-LDHs dispersing in $100 \mathrm{~mL}$ formamide and agitating for $48 \mathrm{~h}$.

2.5. Fabrication of Luminescent Thin Films Based on La-Doped LDHs and Luminescent Thin Films Based on La-Doped LDHs and MMT. PVA was dissolved in deionized water at $90^{\circ} \mathrm{C}$
TABLE 1: Lifetimes of luminescent thin films based on MgAlLaLDHs. $\tau_{1}$ stands for the lifetime of $\operatorname{Mg}(n)$-films and $\tau_{2}$ stands for the lifetime of $\operatorname{MgM}(n)$-films.

\begin{tabular}{|c|c|c|c|c|c|c|}
\hline & \multicolumn{2}{|c|}{8} & \multicolumn{2}{|c|}{16} & \multicolumn{2}{|c|}{24} \\
\hline$\overline{\tau_{i} / \mathrm{ns}}$ & 8.553 & 1.228 & 9.063 & 1.25 & 9.285 & 2.333 \\
\hline$\tau_{1} / \mathrm{ns}$ & \multicolumn{2}{|c|}{8.08} & \multicolumn{2}{|c|}{8.58} & \multicolumn{2}{|c|}{8.98} \\
\hline$\chi^{2}$ & \multicolumn{2}{|c|}{2.768} & \multicolumn{2}{|c|}{2.501} & \multicolumn{2}{|c|}{2.399} \\
\hline$\tau_{i} / \mathrm{ns}$ & 11.95 & 2.463 & 11.51 & 2.644 & 12.62 & 2.933 \\
\hline$\tau_{2} / \mathrm{ns}$ & \multicolumn{2}{|c|}{10.78} & \multicolumn{2}{|c|}{11.37} & \multicolumn{2}{|c|}{12.11} \\
\hline$\chi^{2}$ & \multicolumn{2}{|c|}{3.618} & \multicolumn{2}{|c|}{2.138} & \multicolumn{2}{|c|}{1.183} \\
\hline
\end{tabular}

to form $1 \mathrm{wt} \%$ PVA aqueous solution, and then $1 \mathrm{~g} / \mathrm{L}$ BNMA aqueous solution was mixed with isopyknic PVA aqueous solution to form BNMA@PVA solution.

The quartz slide $\left(3.0 \times 1.0 \mathrm{~cm}^{2}\right)$ was cleaned by the mixture of $\mathrm{NH}_{3} \cdot \mathrm{H}_{2} \mathrm{O}$ and $\mathrm{H}_{2} \mathrm{O}_{2}$ solution and then deposited alternatively in MgAlLa-LDHs suspension and BNMA@PVA solution for $n$ cycles to fabricate (MgAlLa-LDHs/BNMA@ $\mathrm{PVA}_{n}$ (marked as $\mathrm{Mg}(n)$-films). So do (CoAlLa-LDHs/

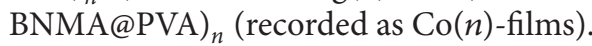

(MMT/BNMA@PVA/MgAlLa-LDHs/BNMA@PVA $)_{n}$, marked as $\operatorname{MgM}(n)$-films, or (MMT/BNMA@PVA/CoAlLaLDHs/BNMA@PVA) $)_{n}$, recorded as $\operatorname{CoM}(n)$-films, were obtained by depositing the MMT suspension, BNMA@PVA solution, LDHs suspension, and BNMA@PVA solution in turn, respectively.

\section{Results and Discussion}

As shown in Figure 1, the intensities of absorption peaks at $265.0 \mathrm{~nm}$ and $372.0 \mathrm{~nm}$ increase linearly during the layers growth, displaying a stepwise and regular growth procedure. The fluorescence emission intensity also displays a consistent increase at the peak in $515.0 \mathrm{~nm}$ with $n$, as shown in Figure 1(a). The luminescent lifetimes of $\mathrm{Mg}(n)$-films (8.08 ns-8.98 ns) are prolonged by a factor of nearly 2.5 times compared with the pristine BNMA powder $(0.37 \mathrm{~ns})$, as shown in Table 1.

This main reason is the rigid structure's isolation effect (IE), which can stop the aggregation of BNMA. The lifetimes of $\mathrm{Mg}(n)$-films are prolonged 1.7 times more than those of (MgAl-LDHs/BNMA@PVS) ${ }_{n}$ (4.61-4.88ns) [18], due to the fact that doping La partially substitutes $\mathrm{Al}$ elements.

As seen from Figure 2, two absorbance peaks of $\operatorname{MgM}(n)$ films are at $265.5 \mathrm{~nm}$ and $375.0 \mathrm{~nm}$, with stable growth with the layer number (Figure 2, inset). Furthermore, at $520.0 \mathrm{~nm}$, the films have the highest emission band peak and are red-shifted by about $5 \mathrm{~nm}$ without any obvious broadening, but the emission intensity also increases regularly with the depositing number. The fluorescence lifetime analysis (Table 1) reveals that the fluorescence lifetime of $\operatorname{MgM}(n)$ films is prolonged by a factor of nearly $30(10.78-12.11 \mathrm{~ns})$ compared with the pristine BNMA powder $(0.37 \mathrm{~ns})$ [18]. Compared with the lifetimes of $\operatorname{Mg}(n)$-films (8.08-8.98 ns), these thin films' lifetimes are also prolonged about $3 \mathrm{~ns}$. The 


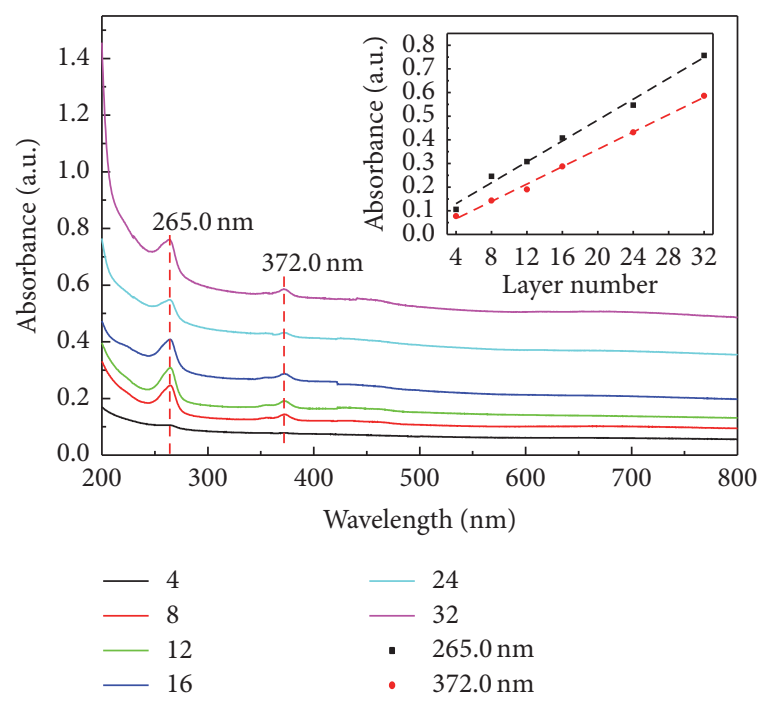

(a)

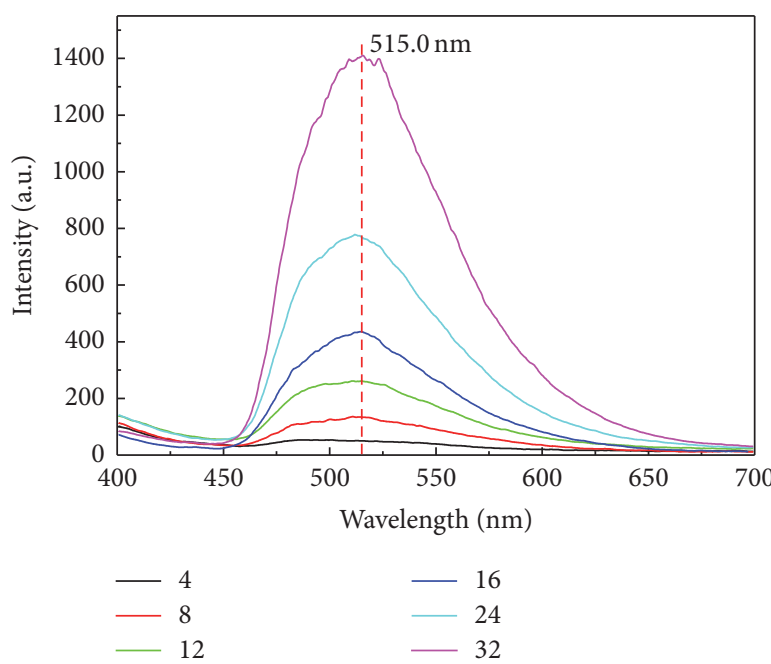

(b)

FIGURE 1: UV-visible absorption spectra and photoluminescence spectra of $\operatorname{Mg}(n)$-films. The inset of (a) shows the absorbance increasing linear relationship in $265.0 \mathrm{~nm}$ and $372.0 \mathrm{~nm}$.

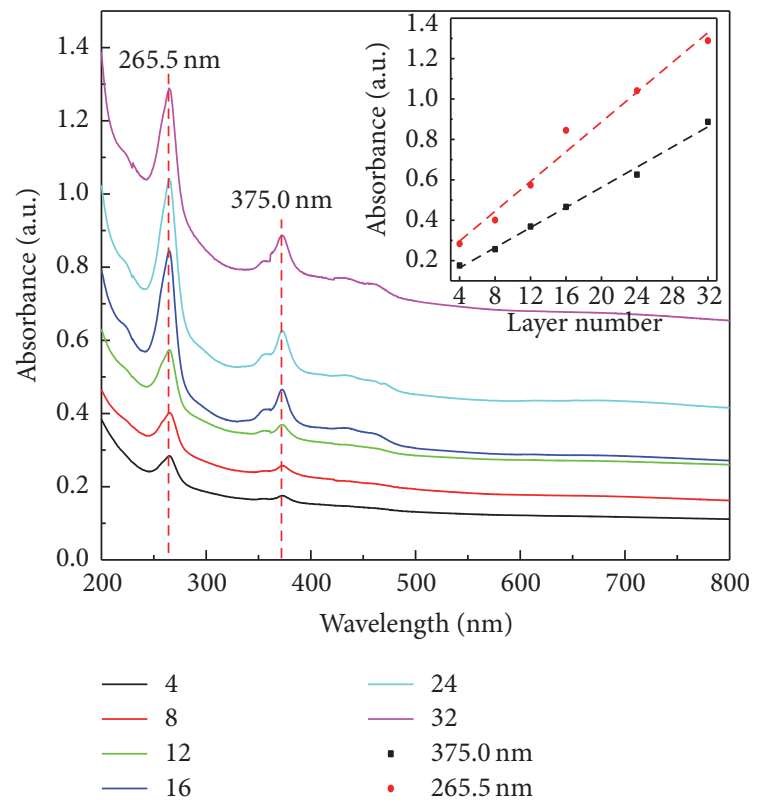

(a)

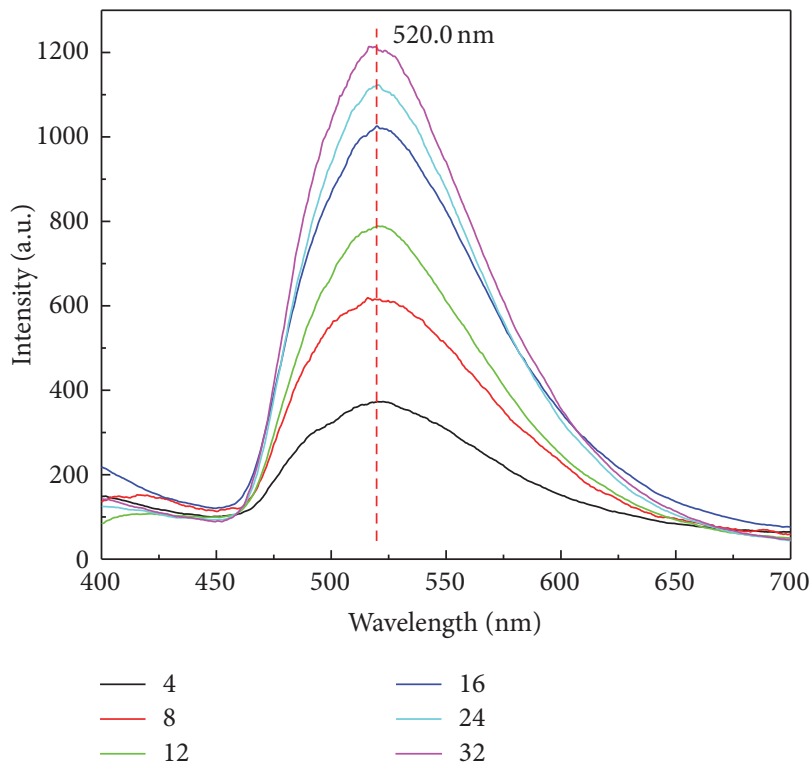

(b)

FIGURE 2: UV-visible absorption spectra and photoluminescence spectra of $\operatorname{MgM}(n)$-films. The inset (a) shows the absorbance increasing linear relationship in $265.5 \mathrm{~nm}$ and $375.0 \mathrm{~nm}$.

first reason is the rigid structure's IE, stopping the aggregation, and formation of BNMA. But the most important one of all is that EM can be formed by the positive LDHs nanosheets and negative MMT nanosheets, which can affect the vibration of backbone so that it can prolong the lifetimes of the films [28].

At the same time, we selected CoAlLa-LDHs and BNMA to assemble the luminescent thin films via LBL method, in order to generate the FE between the interlayers. Photoluminescence spectra of $\mathrm{Co}(n)$-films (Figure 3 ) and ordered and uniform enhanced green luminescent thin films are obtained. Owing to CoAlLa-LDHs offering a constant FE, the luminescent lifetimes of $\mathrm{Co}(n)$-films (9.80-10.52 ns) are over 28 times compared to BNMA powder's lifetimes (0.37 ns) (Table 2), also nearly 3.2 times longer than those of (MgAlLDHs/BNMA@PVS ${ }_{n}(4.61-4.88 \mathrm{~ns})$, and much larger than thin films based on nonmagnetic MgAlLa-LDHs.

Later, we design the nanoarchitecture with the coexistence of EM and FE to explore the thin films' optical properties. In Figure 4, the absorption peaks of BNMA 


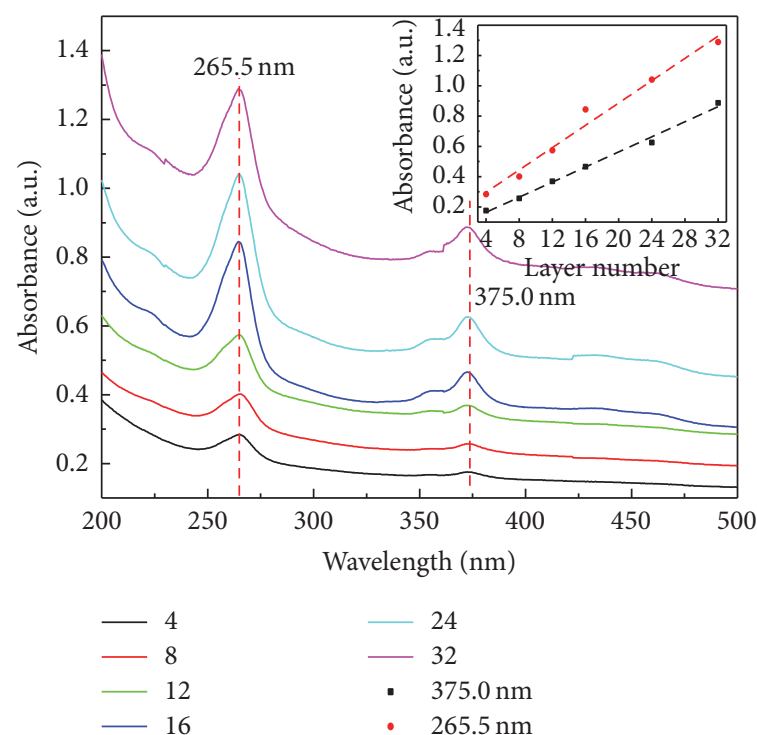

(a)

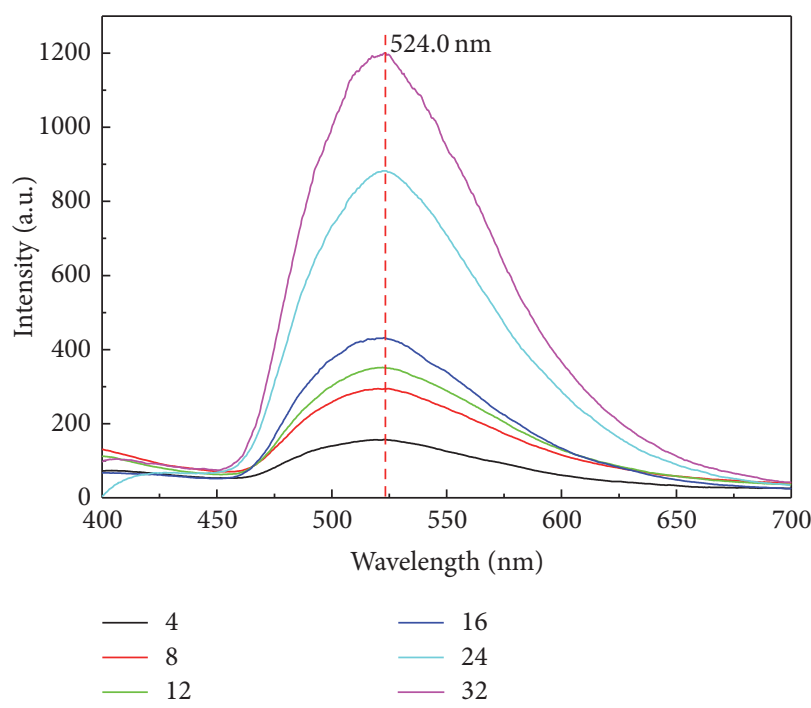

(b)

FIGURE 3: UV-visible absorption spectra and photoluminescence spectra of $\operatorname{Co}(n)$-films. The inset of (a) shows the absorbance increasing linear relationship in $263.5 \mathrm{~nm}, 338.0 \mathrm{~nm}$, and $375.0 \mathrm{~nm}$.

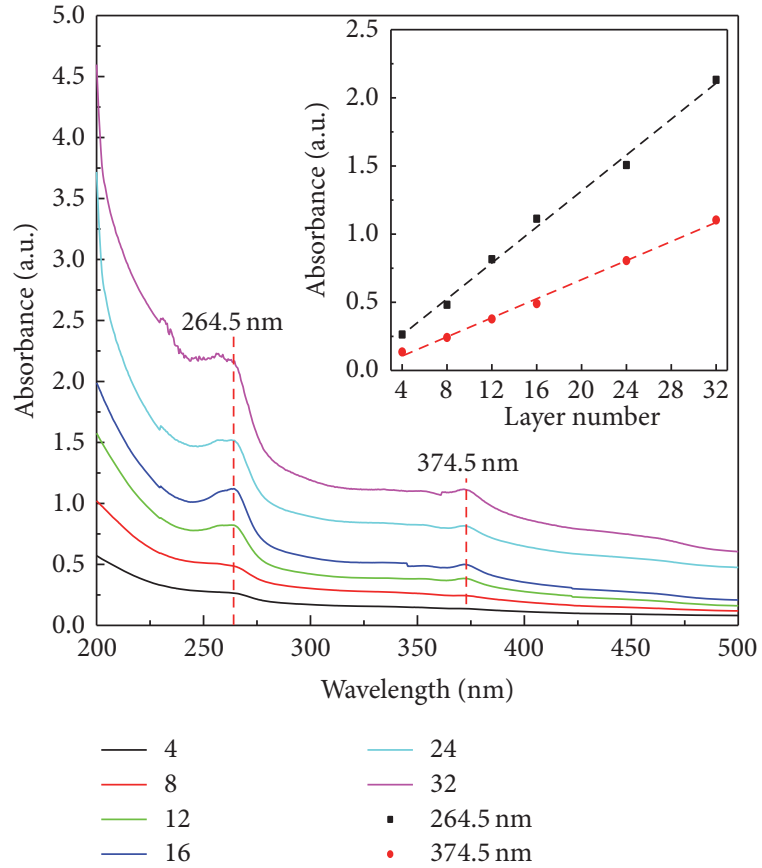

(a)

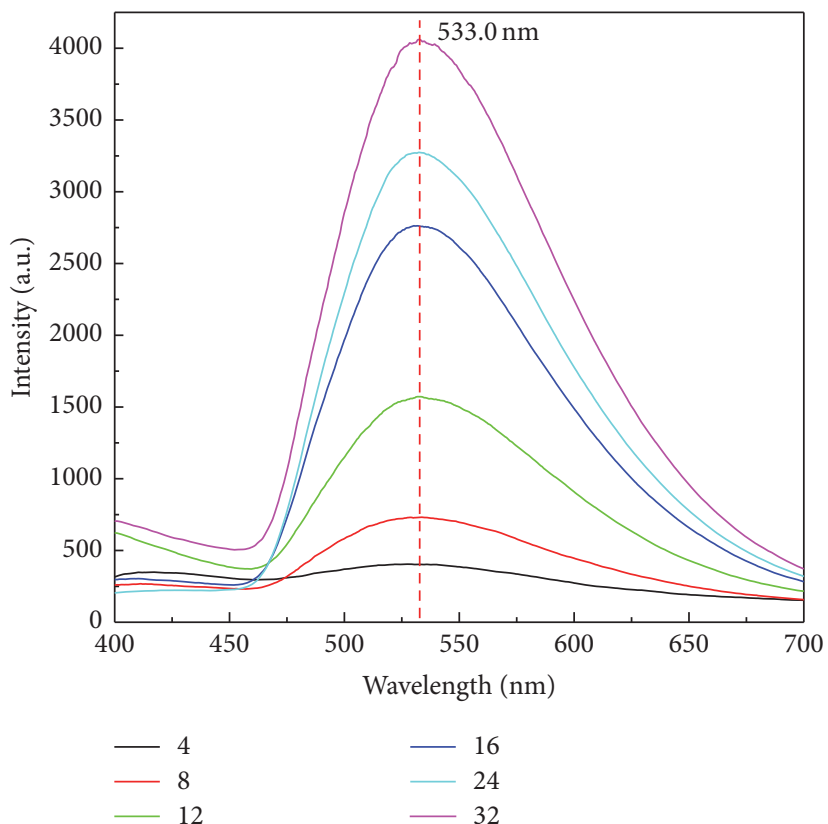

(b)

FIGURE 4: UV-visible absorption spectra and photoluminescence spectra of $\operatorname{CoM}(n)$-films. The inset of (a) shows the absorbance increasing linear relationship in $263.5 \mathrm{~nm}, 338.0 \mathrm{~nm}$, and $375.0 \mathrm{~nm}$.

at $264.5 \mathrm{~nm}$ and $374.5 \mathrm{~nm}$ grow linearly with the cycles number increasing, indicating the films experience wellorganized growth procedure. Besides, the luminescent peak at $533.0 \mathrm{~nm}$ also undergoes a consistent enhancement and is red-shifted by $13 \mathrm{~nm}$ compared with the $\operatorname{MgM}(n)$-films, due to FE generated by CoAlLa-LDHs between the interlayers
[30]. Surprisingly, the luminescent lifetimes of $\mathrm{CoM}(n)$-films (13.67-14.74 ns) are prolonged more than 38-fold than that of BNMA (0.37 ns) and also nearly 4 -fold as long as those of (MgAl-LDHs/BNMA@PVS) $)_{n}$ (4.61-4.88ns) (Table 2). Importantly, the luminescent lifetimes of $\mathrm{CoM}(n)$-films are startlingly extended compared with those of $\operatorname{MgM}(n)$-films. 
TABLE 2: Lifetimes of luminescent thin films based on CoAlLaLDHs. $\tau_{3}$ stands for the lifetime of $\mathrm{Co}(n)$-films and $\tau_{4}$ stands for the lifetime of $\operatorname{CoM}(n)$-films.

\begin{tabular}{|c|c|c|c|c|c|c|}
\hline & \multicolumn{2}{|c|}{8} & \multicolumn{2}{|c|}{16} & \multicolumn{2}{|c|}{24} \\
\hline$\tau_{i} / \mathrm{ns}$ & 10.08 & 2.098 & 10.51 & 1.907 & 10.69 & 2.328 \\
\hline$\tau_{3} / \mathrm{ns}$ & \multicolumn{2}{|c|}{9.80} & \multicolumn{2}{|c|}{10.30} & \multicolumn{2}{|c|}{10.52} \\
\hline$\chi^{2}$ & \multicolumn{2}{|c|}{2.107} & \multicolumn{2}{|c|}{2.279} & \multicolumn{2}{|c|}{2.229} \\
\hline$\overline{\tau_{i} / \mathrm{ns}}$ & 14.02 & 2.707 & 14.54 & 3.743 & 14.97 & 2.983 \\
\hline$\tau_{4} / \mathrm{ns}$ & \multicolumn{2}{|c|}{13.67} & \multicolumn{2}{|c|}{14.05} & \multicolumn{2}{|c|}{14.74} \\
\hline$\chi^{2}$ & \multicolumn{2}{|c|}{1.383} & \multicolumn{2}{|c|}{1.124} & \multicolumn{2}{|c|}{1.208} \\
\hline
\end{tabular}

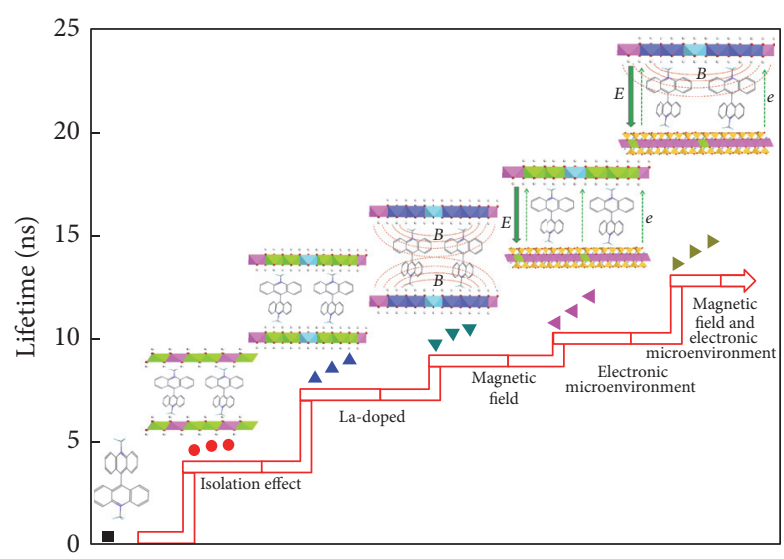

FIGURE 5: The comparison of BNMA's lifetimes in the different states under the different environments. Black dot represents the luminescent lifetime of BNMA powder $(0.37 \mathrm{~ns})$, the red dots stand for those of (MgAl-LDHs/BNMA@PVS ${ }_{n}$, and the blue ones show those of $\operatorname{Mg}(n)$-films. The dark green dots stand for those of $\operatorname{Co}(n)$ films. The pink dots stand for those of $\operatorname{MgM}(n)$-films. The greenyellow dots stand for the luminescent lifetimes of $\operatorname{CoM}(n)$-films.

As a result, it is indicated that CoAlLa-LDHs can affect the luminescent property, thus verifying magnetic effect can prolong the lifetimes of chromophores.

Figure 5 illustrates the comparison for luminescent lifetimes of BNMA in different states under different environments. The lifetimes show stepped increase as the different inorganic nanosheets are introduced. When we only design the doped La nonmagnetic LDHs, the lifetime can markedly increase by nearly 2.8 -fold. But if we choose the doped La magnetic LDHs, the lifetime can prolong over 3 times. When we successfully assemble EM in the nanostructure, the lifetime can extend by $12.11 \mathrm{~ns}$ at most. When introducing La-doped magnetic CoAlLA-LDHs and MMT, the lifetimes reach the highest platform, which obviously confirmed that $\mathrm{EM}$ and FE and the La-doping can prolong luminescent lifetimes, synergistically.

\section{Conclusions}

To sum up, this work successfully assembles the La-doped LDHs and MMT to form EM and designs the cationic chromophores in the rigid microenvironment. Due to the difference of LDHs' components, FE can be introduced in the interlayers. Importantly, it successfully demonstrates that the La-doping, EM, and FE are fairly beneficial to improving the luminescent property. Therefore, it is expected to be potential candidate for manipulating, controlling, and investigating photomagnetoelectric devices.

\section{Competing Interests}

The authors declare that they have no competing interests.

\section{Acknowledgments}

This work was financially supported by the National Natural Science Foundation of China (Grant no., 40802013) and the Fundamental Research Funds for the Central Universities (Grant nos., 2652015092 and 2652015007).

\section{References}

[1] J. P. Liu, Y. Y. Li, X. T. Huang, G. Y. Li, and Z. K. Li, "Layered double hydroxide nano- and microstructures grown directly on metal substrates and their calcined products for application as li-ion battery electrodes," Advanced Functional Materials, vol. 18, no. 9, pp. 1448-1458, 2008.

[2] D. Park, S. Hwang, J. Oh, J. Yang, and J. Choy, "Polymerinorganic supramolecular nanohybrids for red, white, green, and blue applications," Progress in Polymer Science, vol. 38, no. 10-11, pp. 1442-1486, 2013.

[3] G. Hodes, "Perovskite-based solar cells," Science, vol. 342, no. 6156, pp. 317-318, 2013.

[4] F. Song and X. L. Hu, "Exfoliation of layered double hydroxides for enhanced oxygen evolution catalysis," Nature Communications, vol. 5, article 4477, 2014.

[5] V. Nicolosi, M. Chhowalla, M. G. Kanatzidis, M. S. Strano, and J. N. Coleman, "Liquid exfoliation of layered materials," Science, vol. 340, no. 6139, Article ID 1226419, 2013.

[6] T. Lee, S. H. Min, M. Gu et al., "Layer-by-layer assembly for graphene-based multilayer nanocomposites: synthesis and applications," Chemistry of Materials, vol. 27, no. 11, pp. 37853796, 2015.

[7] D. Yan, J. Lu, M. Wei, D. G. Evans, and X. Duan, "Recent advances in photofunctional guest/layered double hydroxide host composite systems and their applications: experimental and theoretical perspectives," Journal of Materials Chemistry, vol. 21, no. 35, pp. 13128-13139, 2011.

[8] J. Bujdák, "Layer-by-layer assemblies composed of polycationic electrolyte, organic dyes, and layered silicates," The Journal of Physical Chemistry C, vol. 118, no. 13, pp. 7152-7162, 2014.

[9] V. Nicolosi, M. Chhowalla, M. G. Kanatzidis, and M. S. Strano, "Dispersible exfoliated zeolite nanosheets and their application as a selective membrane," Science, vol. 334, no. 6052, pp. 72-75, 2011.

[10] D.-M. Xu, M.-Y. Guan, Q.-H. Xu, and Y. Guo, "Multilayer films of layered double hydroxide/polyaniline and their ammonia sensing behavior," Journal of Hazardous Materials, vol. 262, pp. 64-70, 2013.

[11] Y. Y. Zhong, Y. Q. Liao, A. M. Gao et al., "Supercapacitive behavior of electrostatic self-assembly reduced graphene oxide/CoAllayered double hydroxides nanocomposites," Journal of Alloys and Compounds, vol. 669, pp. 146-155, 2016. 
[12] Y. Guo, X. Liu, X. Wang et al., "Carbon dot/NiAl-layered double hydroxide hybrid material: facile synthesis, intrinsic peroxidase-like catalytic activity and its application," RSC Advances, vol. 5, no. 116, pp. 95495-95503, 2015.

[13] W. Shi, Y. Jia, S. Xu et al., "A chiroptical switch based on DNA/layered double hydroxide ultrathin films," Langmuir, vol. 30, no. 43, pp. 12916-12922, 2014.

[14] D. Chen, X. Wang, T. Liu, X. Wang, and J. Li, "Electrically conductive poly(vinyl alcohol) hybrid films containing graphene and layered double hydroxide fabricated via layer-by-layer selfassembly," ACS Applied Materials \& Interfaces, vol. 2, no. 7, pp. 2005-2011, 2010.

[15] L. Li, R. Z. Ma, Y. Ebina, K. Fukuda, K. Takada, and T. Sasaki, "Layer-by-layer assembly and spontaneous flocculation of oppositely charged oxide and hydroxide nanosheets into inorganic sandwich layered materials," Journal of the American Chemical Society, vol. 129, no. 25, pp. 8000-8007, 2007.

[16] D. Yan, J. Lu, L. Chen et al., "A strategy to the ordered assembly of functional small cations with layered double hydroxides for luminescent ultra-thin films," Chemical Communications, vol. 46, no. 32, pp. 5912-5914, 2010.

[17] K. Binnemans, "Lanthanide-based luminescent hybrid materials,” Chemical Reviews, vol. 109, no. 9, pp. 4283-4374, 2009.

[18] J. Zhou, G. Chen, Y. Zhu et al., "Intense multiphoton upconversion of $\mathrm{Yb}^{3+}-\mathrm{Tm}^{3+}$ doped $\beta-\mathrm{NaYF}_{4}$ individual nanocrystals by saturation excitation," Journal of Materials Chemistry C, vol. 3, no. 2, pp. 364-369, 2015.

[19] H. Suo, C. Guo, Z. Yang, S. Zhou, C. Duan, and M. Yin, "Thermometric and optical heating bi-functional properties of upconversion phosphor $\mathrm{Ba}_{5} \mathrm{Gd}_{8} \mathrm{Zn}_{4} \mathrm{O}_{21}: \mathrm{Yb}^{3+} / \mathrm{Tm}^{3+}$," Journal of Materials Chemistry C, vol. 3, no. 28, pp. 7379-7385, 2015.

[20] Z. Mao, J. Chen, L. Sun, Q. Lu, and D. Wang, "Control of green emitting intermediate phases in $(\mathrm{Ba}, \mathrm{Sr})_{3} \mathrm{MgSi}_{2} \mathrm{O}_{8}: \mathrm{Eu}^{2+}, \mathrm{Mn}^{2+}$ phosphors for dual-bands or tri-bands emission," Materials Research Bulletin, vol. 70, pp. 908-913, 2015.

[21] S. Gago, M. Pillinger, R. A. Sá Ferreira, L. D. Carlos, T. M. Santos, and I. S. Gonçalves, "Immobilization of lanthanide ions in a pillared layered double hydroxide," Chemistry of Materials, vol. 17, no. 23, pp. 5803-5809, 2005.

[22] P. Gunawan and R. Xu, "Lanthanide-doped layered double hydroxides intercalated with sensitizing anions: efficient energy transfer between host and guest layers," The Journal of Physical Chemistry C, vol. 113, no. 39, pp. 17206-17214, 2009.

[23] X. Dong, L. Wang, D. Wang, C. Li, and J. Jin, "Layer-by-layer engineered Co-Al hydroxide nanosheets/graphene multilayer films as flexible electrode for supercapacitor," Langmuir, vol. 28, no. 1, pp. 293-298, 2012.

[24] H. Zhang, G. Zhang, X. Bi, and X. Chen, "Facile assembly of a hierarchical core@shell $\mathrm{Fe}_{3} \mathrm{O}_{4} @ \mathrm{CuMgAl}-\mathrm{LDH}$ (layered double hydroxide) magnetic nanocatalyst for the hydroxylation of phenol," Journal of Materials Chemistry A, vol. 1, no. 19, pp. 5934-5942, 2013.

[25] M. F. Shao, J. B. Han, W. Y. Shi, M. Wei, and X. Duan, "Layer-bylayer assembly of porphyrin/layered double hydroxide ultrathin film and its electrocatalytic behavior for $\mathrm{H}_{2} \mathrm{O}_{2}$, Electrochemistry Communications, vol. 12, no. 8, pp. 1077-1080, 2010.

[26] H. Chen, L. Hu, M. Chen, Y. Yan, and L. Wu, "Nickel-cobalt layered double hydroxide nanosheets for high-performance supercapacitor electrode materials," Advanced Functional Materials, vol. 24, no. 7, pp. 934-942, 2014.

[27] M. Ikeda, T. Yoshii, T. Matsui, T. Tanida, H. Komatsu, and I. Hamachi, "Montmorillonite-supramolecular hydrogel hybrid for fluorocolorimetric sensing of polyamines," Journal of the American Chemical Society, vol. 133, no. 6, pp. 1670-1673, 2011.

[28] T. Wang, M. Liu, H. Ma, X. Liu, Y. Fu, and K. Hu, "Lifetimeultra-prolonged luminescent multilayer thin films with electronic microenvironment," RSC Advances, vol. 4, no. 77, pp. 40748-40752, 2014.

[29] M. Liu, T. Wang, H. Ma, Y. Fu, K. Hu, and C. Guan, "Assembly of luminescent ordered multilayer thin-films based on oppositelycharged MMT and magnetic NiFe-LDHs nanosheets with ultralong lifetimes," Scientific Reports, vol. 4, article no. 7147, 2014.

[30] N. C. Bigall, W. J. Parak, and D. Dorfs, "Fluorescent, magnetic and plasmonic-hybrid multifunctional colloidal nano objects," Nano Today, vol. 7, no. 4, pp. 282-296, 2012. 

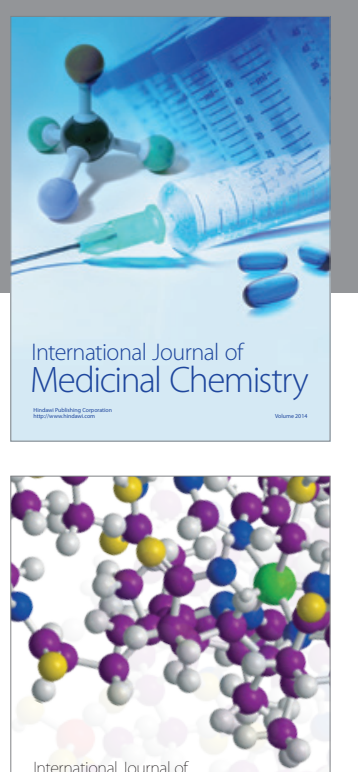

Carbohydrate Chemistry

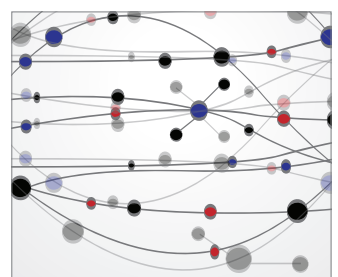

The Scientific World Journal
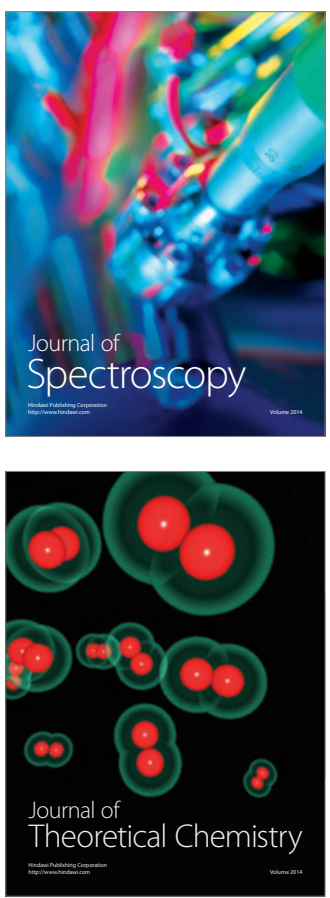
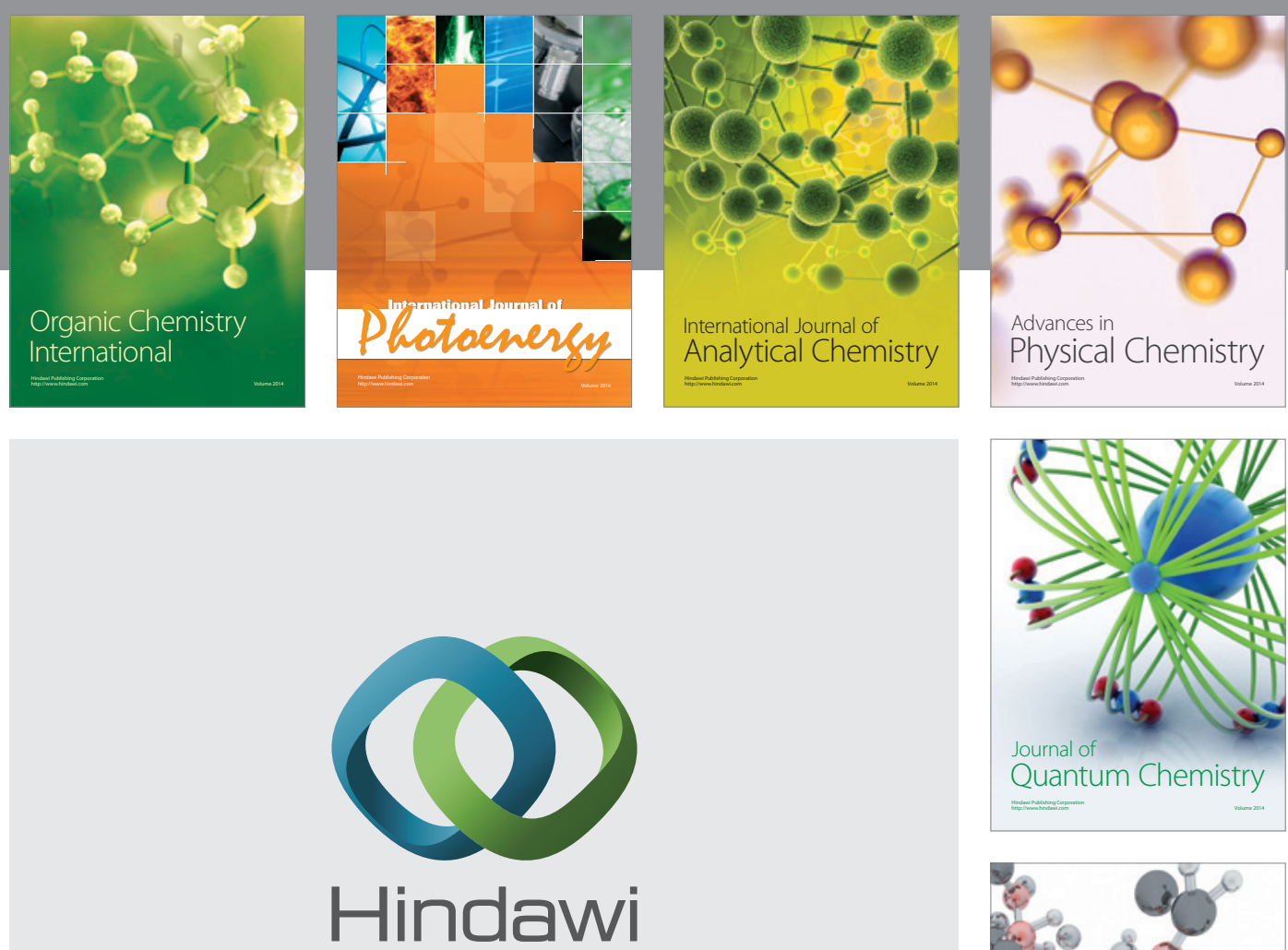

Submit your manuscripts at

https://www.hindawi.com

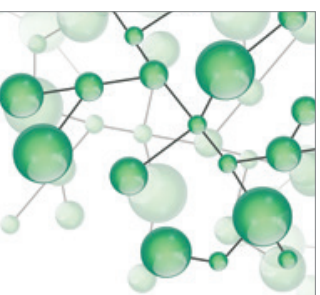

International Journal of

Inorganic Chemistry
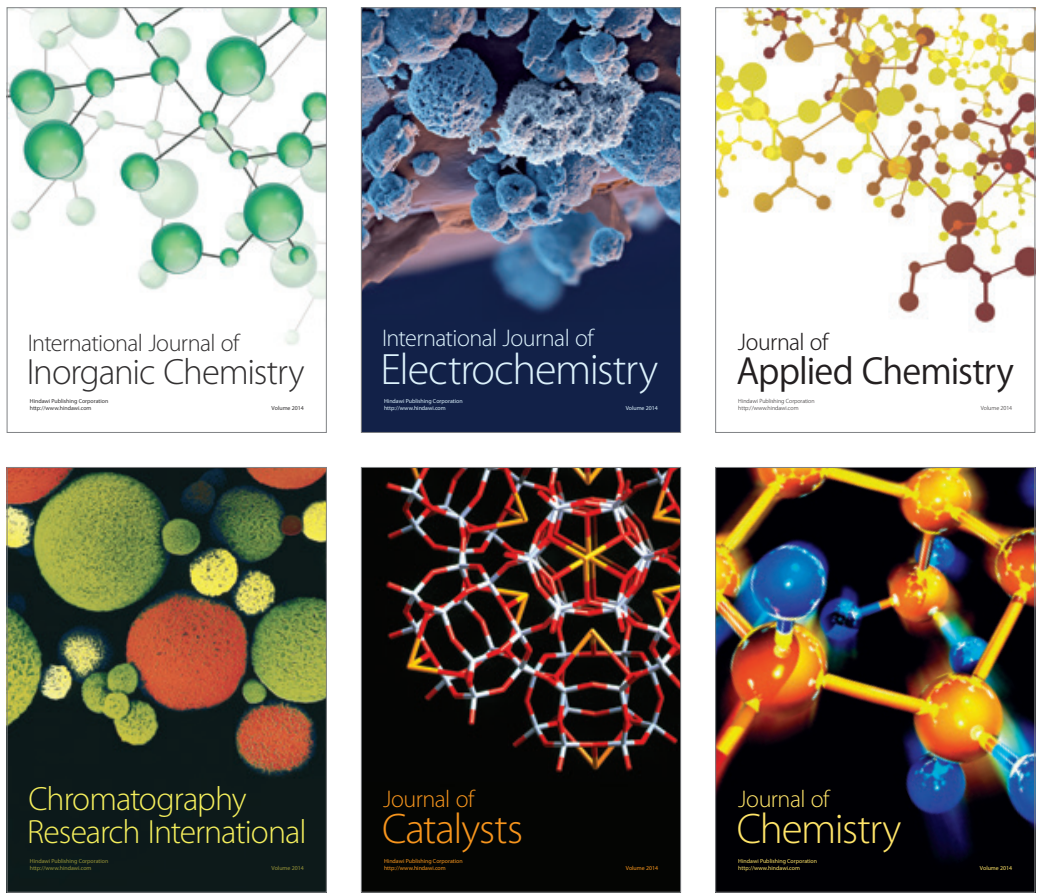

Journal of

Applied Chemistry
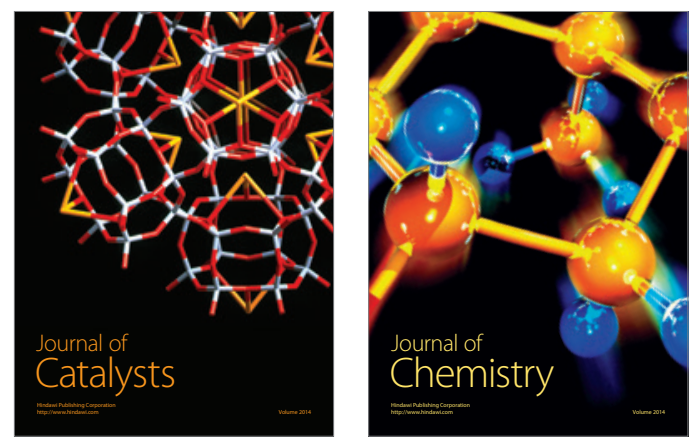
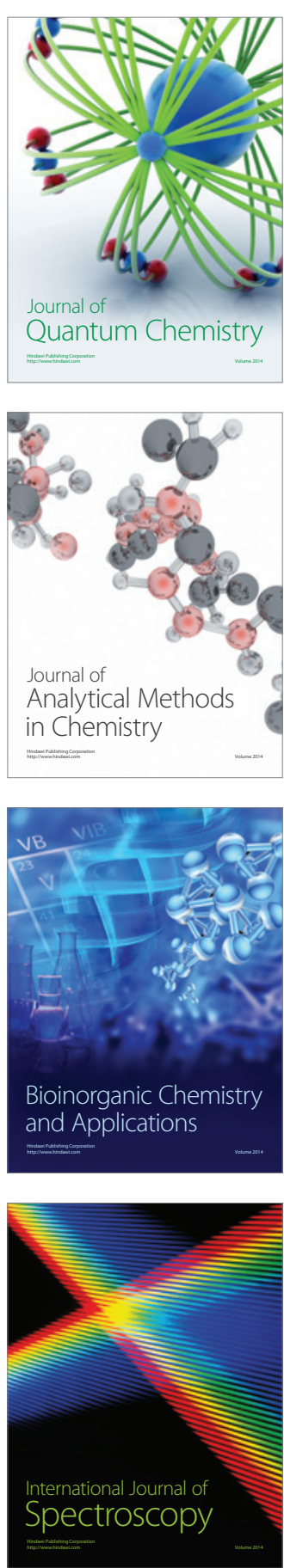